\title{
Combined Analytical/FEM Approach for Efficient Simulation of Lamb Wave Damage Detection
}

\author{
Yanfeng Shen ${ }^{1}$, Victor Giurgiutiu ${ }^{2}$ \\ ${ }^{1}$ Department of Aerospace Engineering, University of Michigan, USA, 48105 \\ ${ }^{2}$ Department of Mechanical Engineering, University of South Carolina, USA, 29208
}

\begin{abstract}
Lamb waves have been widely explored as a promising inspection tool for non-destructive evaluation (NDE) and structural health monitoring (SHM). This article presents a combined analytical/finite element model (FEM) approach (CAFA) for the accurate, efficient, and versatile simulation of 2-D Lamb wave propagation and interaction with damage. CAFA used a global analytical solution to model wave generation, propagation, scattering, mode conversion, and detection, while the wave-damage interaction coefficients (WDICs) were extracted from harmonic analysis of local FEM with non-reflective boundaries (NRB). The analytical procedure was coded using MATLAB, and a predictive simulation tool called WaveFormRevealer 2-D was developed. The methodology of obtaining WDICs from local FEM was presented. Case studies were carried out for Lamb wave propagation in a pristine plate and a damaged plate. CAFA predictions compared well with full scale multi-physics FEM simulations and experiments with scanning laser Doppler vibrometry (SLDV), while achieving remarkable performance in computational efficiency and computer resource saving compared with conventional FEM.
\end{abstract}

\section{INTRODUCTION}

The development of computational models for Lamb wave propagation and interaction is of great importance for SHM system design and sensing signal interpretation. Effective design of SHM systems requires the exploration of a wide range of parameters (transducer size, sensor-damage relative locations, interrogating wave characteristics, etc.) to achieve best detection and quantification of certain types of damage. On the other hand, active sensing signals using Lamb waves are usually hard to interpret due to the multi-mode, dispersive nature of Lamb waves and their interaction with damage, involving complicated scattering and mode conversion phenomena. Practical applications have imposed three main requirements on the computational models for Lamb wave propagation: (1) accuracy for high frequency, short wavelength, and long propagation distance waves; (2) efficiency in terms of computational time and computer resources; (3) versatility for exploring a wide range of design parameters. However, commercial simulation tools cannot satisfy all these requirements.

\subsection{Piezoelectric Wafer Active Sensors}

In this study, we used the piezoelectric wafer active sensors (PWAS) as an enabling device for 
generating Lamb waves in the host structures. PWAS have been widely used in SHM for generating and receiving ultrasonic waves [1]. They couple the electrical and mechanical effects (mechanical strain, $S_{i j}$, mechanical stress, $T_{k l}$, electrical field, $E_{k}$, and electrical displacement, $D_{j}$ ) through the tensorial piezoelectric constitutive equations

$$
\begin{aligned}
& S_{i j}=s_{i j k l}^{E} T_{k l}+d_{k i j} E_{k} \\
& D_{j}=d_{j k l} T_{k l}+\varepsilon_{j k}^{T} E_{k}
\end{aligned}
$$

where $s_{i j k l}^{E}$ is the mechanical compliance of the material measured at zero electric field $(E=0), \varepsilon_{j k}^{T}$ is the dielectric permittivity measured at zero mechanical stress $(T=0)$, and $d_{j k l}$ represents the piezoelectric coupling effect. PWAS utilize the $d_{31}$ coupling between in-plane strains, $S_{1}, S_{2}$ and transverse electric field $E_{3}$.

Compared with conventional ultrasonic transducers, PWAS are small, lightweight, low cost, and unobtrusive. They can be permanently bonded on host structures and achieve real time active sensing and in-situ monitoring. PWAS can serve several purposes: (a) high-bandwidth strain sensors; (b) high-bandwidth wave exciters and receivers; (c) resonators; (d) embedded modal sensors with the electromechanical (E/M) impedance method. By application types, PWAS transducers can be used for (i) active sensing of far-field damage using pulse-echo, pitch-catch, and phased-array methods, (ii) active sensing of near field damage using high-frequency E/M impedance method and thickness gage mode, and (iii) passive sensing of damage-generating events through detection of low-velocity impacts and acoustic emission at the tip of advancing cracks.

\subsection{STATE OF THE ART}

Research has been conducted on analytical models of PWAS generated Lamb waves and the tuning effect between PWAS transducers and host structures [1, 2]. These models only consider the wave propagation in pristine structures, whereas the design of SHM systems requires research into wave-damage interaction. In our previous study, we proposed an analytical parametric model for 1-D Lamb wave propagation with damage effects, but this model has two limitations: (1) 1-D formulation cannot accurately capture the 2-D outward propagation pattern in practical applications; (2) the WDICs cannot be determined quantitatively [3].

Regarding the aspect of wave-damage interaction, many researchers have developed analytical models using Kirchhoff, Mindlin, Kane-Mindlin plate theory, and 3-D elasticity 
solution or exact Lamb mode solutions $[4,5,6,7,8,9,10,11]$, while others have adopted numerical methods such as finite element method (FEM) $[12,13]$, boundary element method (BEM) $[14,15]$, spectral element method (SEM) [16], and finite difference method (LISA) $[17,18]$. The advantage of analytical models is that they are accurate, efficient, and capable of providing fast parametric studies, but the drawback is that they only apply to simple damage geometries such as circular holes, partial-through circular holes, or flat-bottom cavities. The advantage of numerical methods is that they can simulate wave damage interaction phenomena in very complicated structures; however, these methods usually require the discretization of the analyzed domain and the time marching procedure. For such high frequency, short wavelength, and long propagation wave simulations, considerably small time marching step and dense discretization are required to obtain an accurate solution, which can make the target problem computationally prohibitive.

To make the computational load manageable, small-size numerical methods have been developed. Distributed point source method (DPSM) calculates wave generation and scattering in a highly efficient manner $[19,20]$. But one needs to evaluate the complicated Green's matrices of a dynamic system. Hybrid modeling techniques have been proposed to develop efficient simulation schemes. The semi-analytical finite element (SAFE) method has been used to combine with local FEM to simulate wave interaction with damage in 1-D wave propagation problems, but 2-D Lamb wave propagation models have not been achieved [21, 22 , 23]. Promising results by combining analytical solution with local FEM have been achieved for bulk wave scattering from an axisymmetric inclusion and Lamb wave interaction with cracks in rivet holes [24, 25]. Terrien et al. investigated the optimization of hidden corrosion detection in aircraft structures using Lamb waves [26]. In their study, they combined a local 2-D FEM with global 1-D wave modal decomposition to predict the structural response. However, these studies considered only bulk waves or single symmetric Lamb mode interaction with damage, and used conventional wedge transmitters and bulky accelerometers as receivers to pick up the out-of-plane acceleration associated with the wave motion. They further require the solution matching at each node at the local FEM boundary, which demands specialist finite element codes, making them hard to benefit wide engineering applications. Moreau and Castaings have used orthogonally relation to reduce the size of FEM to obtain 3-D guided wave scattering features [27]. Gresil and Giurgiutiu investigated the hybrid analytical/FEM modeling concept in time domain, and achieved promising results $[28,29]$. Obenchain et al. used a hybrid global matrix/LISA for modeling wave propagation in composites [30]. Zhang et al. used the hybrid modeling concept to model the performance of ultrasonic arrays for bulk wave inspection [31]. Velichko and Wilcox applied similar hybrid approach to model guided wave propagation and scattering in pipes, constructing an inverse problem to post-process guided wave array data for damage imaging [32]. Recently, the development of local FEM techniques allows us to conveniently study the ultrasonic scattering and mode conversion phenomena in bulk wave and guided waves from complicated 3-D damage [33, 34, 35, 36, 37]. 
In this study, we developed a Combined Analytical/FEM Approach (CAFA) for the special treatment of efficiently simulating PWAS-generated 2-D Lamb wave propagation and interaction with damage. Admittedly, the analytical model of 2-D guided wave generation and propagation has been reported in a number of literatures in the form of time-harmonic solution $[1,2]$. However, the illustration of detailed steps to obtain the time domain solutions under an arbitrary excitation and the insertion of damage effects into such PWAS-generated 2-D Lamb wave analytical model has not been systematically addressed in current literatures. We are not trying to compare with other methods such as DPSM, LISA and SAFE; instead, we aim to combine mature techniques such as the analytical approach and the local FEM into a complete and systematic model. The ultimate goal is to obtain fast predictions of time-space domain full field sensing signals and achieve efficient SHM system design. Further, we developed WaveFormRevealer 2-D as a user-friendly simulation tool for the realization of CAFA.

\section{OVERVIEW OF CAFA}

Figure 1 shows the schematic for CAFA. Lamb wave generation, propagation, damage interaction (scattering, mode conversion), and detection are modeled using the exact analytical expressions, while the wave damage interaction coefficients (WDICs) are extracted from the harmonic analysis of small-size local FEM with NRB. CAFA couples the global analytical wave expression with the local FEM solution through WDICs. It combines the virtues of both analytical and numerical methods: the analytical formulation provides high calculation efficiency, a wide parameter exploration capability, and excellent accuracy, while FEM has the ability to simulate damage with complicated geometries.

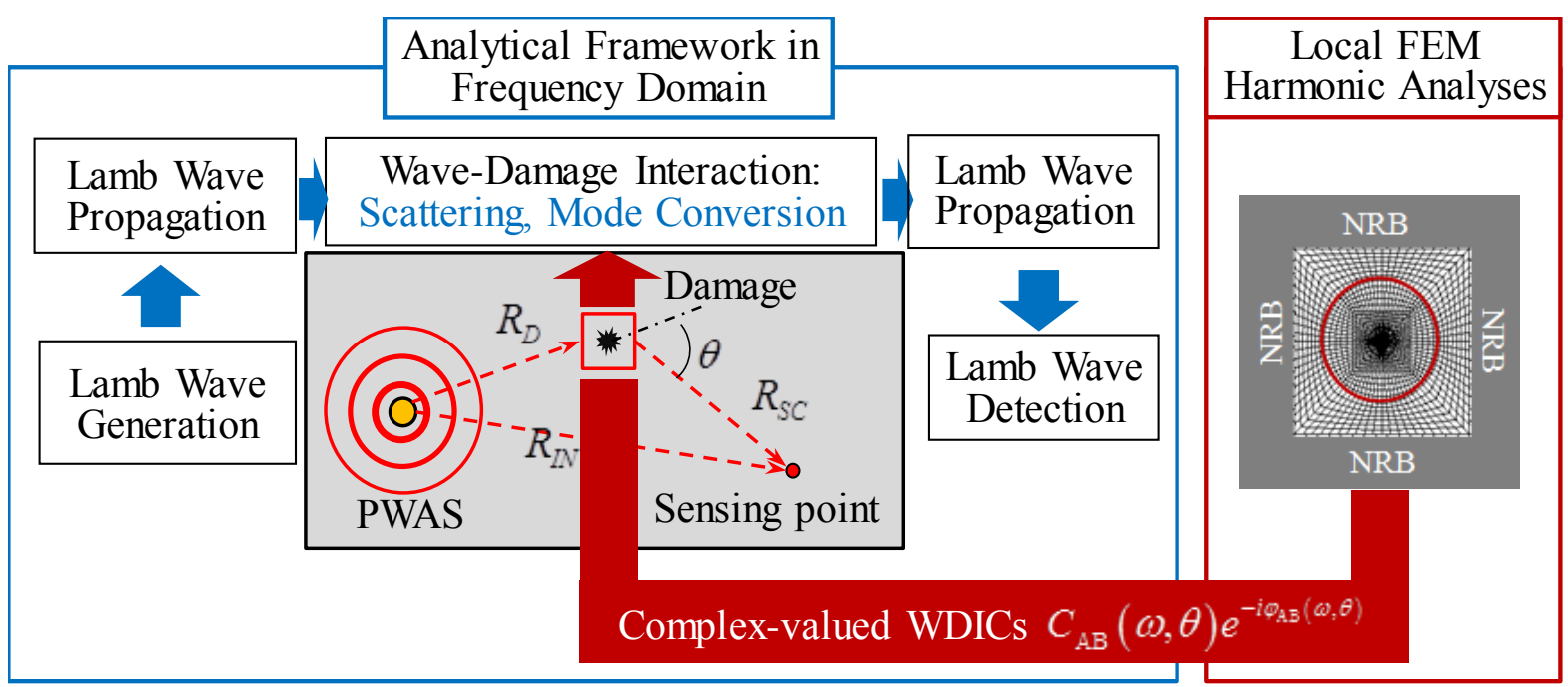

Figure 1: Overview of Combined Analytical/FEM Approach (CAFA). 


\section{ANALYTICAL FrameWORK FOR LAMB WAVE ACTIVE SENSING}

In this section, we will present the methodology for modeling damage effects, and the steps required to construct the analytical framework for PWAS-generated 2-D Lamb wave propagation and interaction with damage.

\subsection{Modeling of Damage EfFects Using CompleX-VAlued WDIC}

In an active sensing approach using PWAS and scanning laser Doppler vibrometry (SLDV), the transmitter PWAS (T-PWAS) generates Lamb waves into the host structure under electrical excitation. Lamb waves propagate along the structure, interact with the damage, undergo scattering and mode conversion, and are finally picked up by the SLDV, which measures the out-of-plane particle velocity at the surface of the structure.

In the analytical framework, the received signal is comprised of two parts: (1) direct incident waves from the T-PWAS; (2) scattered waves from the damage. Thus, the damage can be modeled as a secondary wave source. The total wave field $W_{\text {TOTAL }}$ is the superposition of the

incident wave field $W_{I N}$ and the scattered wave field $W_{S C}$ from the damage.

$$
W_{\text {TOTAL }}=W_{I N}+W_{S C}
$$

We used frequency and direction dependent complex-valued WDICs $C_{\mathrm{AB}}(\omega, \theta) e^{-i \varphi_{\mathrm{AB}}(\omega, \theta)}$ to model the scattering and mode conversion phenomena of wave-damage interaction. These WDICs are capable of describing the amplitude and phase of the scattered waves as a function of frequency and direction. Our notations are as follows: two letters are used to describe the interaction phenomena, with the first letter $A$ denoting the incident wave type, and the second letter $B$ standing for the resulting wave type. For instance, SS (symmetric-symmetric) means the incident symmetric waves are scattered as symmetric waves, SA (symmetric-antisymmetric) means incident symmetric waves are scattered and mode converted to antisymmetric waves, and SSH (symmetric-shear horizontal) means incident symmetric waves are scattered and mode converted to shear horizontal waves. Thus, WDIC $C_{S S}(\omega, \theta) e^{-i \varphi_{S S}(\omega, \theta)}$ denotes the scattered symmetric mode generated by incident symmetric mode with amplitude ratio $C_{S S}(\omega, \theta)$ and phase shift $\varphi_{S S}(\omega, \theta)$. Similarly, $C_{S A}(\omega, \theta) e^{-i \varphi_{S A}(\omega, \theta)}$ represents the scattered antisymmetric mode generated by incident symmetric mode with amplitude ratio $C_{S A}(\omega, \theta)$ and phase shift $\varphi_{S A}(\omega, \theta)$. $C_{S S H}(\omega, \theta) e^{-i \varphi_{S S H}(\omega, \theta)}$ stands for the scattered shear horizontal mode generated by incident 
symmetric mode with amplitude ratio $C_{S S H}(\omega, \theta)$ and phase shift $\varphi_{S S H}(\omega, \theta) . \omega$ is the wave component frequency and $\theta$ represents the scattering angle with respect to the incident wave direction. These coefficients are determined by damage features and are calculated from the small-size local FEM harmonic analysis, which will be introduced later.

\subsection{2-D ANALYTICAL FRAMEWORK CONSTRUCTION}

The analytical model was constructed in frequency domain based on the exact 2-D Lamb wave solution in the following steps:

STEP 1: Perform Fourier transform of the time-domain excitation signal $V_{T}(t)$ to obtain the frequency domain excitation spectrum, $\tilde{V}_{T}(\omega)$.

STEP 2: Calculate structure transfer function. Detailed analytical derivation of 2-D Lamb waves generated by a circular transmitter PWAS is given in Ref [1].

$$
\begin{aligned}
\left.u_{r}(r, t ; \omega)\right|_{z=d} & =-\pi i \frac{a^{2} \tau_{a}}{2 \mu} \sum_{\xi^{S}} \frac{J_{1}\left(\xi^{S} a\right) N_{S}\left(\xi^{S}\right)}{D_{S}^{\prime}\left(\xi^{S}\right)} H_{1}^{(1)}\left(\xi^{S} r\right) e^{-i \omega t} \\
& -\pi i \frac{a^{2} \tau_{a}}{2 \mu} \sum_{\xi^{A}} \frac{J_{1}\left(\xi^{A} a\right) N_{A}\left(\xi^{A}\right)}{D_{A}^{\prime}\left(\xi^{A}\right)} H_{1}^{(1)}\left(\xi^{A} r\right) e^{-i \omega t}
\end{aligned}
$$

In Eq. (3), the frequency $\omega$ acts as a parameter. The component functions of Eq. (3) are defined as

$$
\begin{aligned}
& N_{S}(\xi)=\xi \eta_{S}\left(\xi^{2}+\eta_{S}^{2}\right) \cos \eta_{P} d \cos \eta_{S} d \\
& N_{A}(\xi)=-\xi \eta_{S}\left(\xi^{2}+\eta_{S}^{2}\right) \sin \eta_{P} d \sin \eta_{S} d \\
& D_{S}=\left(\xi^{2}-\eta_{S}^{2}\right)^{2} \cos \eta_{P} d \sin \eta_{S} d+4 \xi^{2} \eta_{P} \eta_{S} \sin \eta_{P} d \cos \eta_{S} d \\
& D_{A}=\left(\xi^{2}-\eta_{S}^{2}\right)^{2} \sin \eta_{P} d \cos \eta_{S} d+4 \xi^{2} \eta_{P} \eta_{S} \cos \eta_{P} d \sin \eta_{S} d
\end{aligned}
$$

where $a$ is the radius of the circular PWAS, $d$ is half plate thickness, and $r$ is the distance between the point of interest and T-PWAS. $\tau_{a}$ represents shear stress between the transducer

and host structure and $\mu$ denotes the shear modulus of the structure. $J_{1}$ is Bessel function of order one, which captures the tuning effect between PWAS and the host structure. $H_{1}^{(1)}$ is the Hankel function of the first kind and order one, which represents an outward propagating 2-D wave field. $\xi$ is the frequency dependent wavenumber calculated from the Rayleigh-Lamb equation $[38,39]$ : 


$$
\begin{gathered}
\frac{\tan \eta_{S} d}{\tan \eta_{P} d}=\left[\frac{-4 \eta_{P} \eta_{S} \xi^{2}}{\left(\xi^{2}-\eta_{S}^{2}\right)^{2}}\right]^{ \pm 1} \\
\eta_{P}^{2}=\frac{\omega^{2}}{c_{P}^{2}}-\xi^{2} ; \quad \eta_{S}^{2}=\frac{\omega^{2}}{c_{S}^{2}}-\xi^{2} ; \quad c_{P}=\sqrt{\frac{\lambda+2 \mu}{\rho}} ; \quad c_{S}=\sqrt{\frac{\mu}{\rho}}
\end{gathered}
$$

where +1 exponent corresponds to symmetric Lamb wave modes and -1 exponent corresponds to antisymmetric Lamb wave modes. $c_{P}$ and $c_{S}$ represent the P-wave speed and S-wave speed; $\lambda$ is Lame's constant of the structural material; $\rho$ is the material density. The transfer function of the transducer, $\kappa_{P W A S}(\omega)$, which converts the applied voltage $\tilde{V}_{T}(\omega)$ into the shear stress $\tau_{a}$, is given by

$$
\kappa_{P W A S}(\omega)=\frac{d_{31}}{s_{11}^{E}} \frac{r(\omega)}{1-r(\omega)}
$$

where $r(\omega)$ is the stiffness ratio between host structure and T-PWAS [40].

At a given excitation frequency $\omega$, Eq. (5) yields a multitude of symmetric (S) and antisymmetric (A) wavenumbers $\xi^{S}, \xi^{A}$ which are used in calculating the summations contained in Eq. (3). Substitution of Eq. (6) into Eq. (3) and division by $e^{-i \omega t}$ yields the structural transfer function that can be separated, for convenience, into $\mathrm{S}$ and A parts, i.e.,

$$
\begin{aligned}
& G^{S}(\omega, r)=-\pi i \frac{a^{2} \kappa_{P W A S}(\omega)}{2 \mu} \sum_{\xi^{S}} \frac{J_{1}\left(\xi^{S} a\right) N_{S}\left(\xi^{S}\right)}{D_{S}^{\prime}(\xi)} H_{1}^{(1)}\left(\xi^{S} r\right) \\
& G^{A}(\omega, r)=-\pi i \frac{a^{2} \kappa_{P W A S}(\omega)}{2 \mu} \sum_{\xi^{A}} \frac{J_{1}\left(\xi^{A} a\right) N_{A}\left(\xi^{A}\right)}{D_{A}^{\prime}(\xi)} H_{1}^{(1)}\left(\xi^{A} r\right)
\end{aligned}
$$

STEP 3: Multiply the structure transfer function by the frequency-domain excitation signal to obtain the direct incident waves at the sensing location, where the distance $R_{I N}$ from PWAS up to sensing location is used. Similarly, multiply the structure transfer function up to the damage location by the frequency-domain excitation signal to obtain the interrogating waves arriving at the damage, where the distance $R_{D}$ from PWAS up to the damage location is used. 


$$
\begin{gathered}
u_{I N}\left(\omega, R_{I N}\right)=\tilde{V}_{T}(\omega)\left[G^{S}\left(\omega, R_{I N}\right)+G^{A}\left(\omega, R_{I N}\right)\right] \\
u_{D}\left(\omega, R_{D}\right)=\tilde{V}_{T}(\omega)\left[G^{S}\left(\omega, R_{D}\right)+G^{A}\left(\omega, R_{D}\right)\right]
\end{gathered}
$$

It can be noticed that the Lamb modes propagate independently and the direct incident wave field is the superposition of each wave mode.

STEP 4: Scattering wave source at the damage location is obtained by modifying incident waves at the damage with WDICs.

$$
\begin{gathered}
u_{N}^{S}=C_{S S}(\omega, \theta) e^{-i \varphi_{S S}(\omega, \theta)} u_{D}^{S}+C_{A S}(\omega, \theta) e^{-i \varphi_{A S}(\omega, \theta)} u_{D}^{A} \\
u_{N}^{A}=C_{S A}(\omega, \theta) e^{-i \varphi_{S A}(\omega, \theta)} u_{D}^{S}+C_{A A}(\omega, \theta) e^{-i \varphi_{A A}(\omega, \theta)} u_{D}^{A} \\
u_{N}^{S H}=C_{S S H}(\omega, \theta) e^{-i \varphi_{S S H}(\omega, \theta)} u_{D}^{S}+C_{A S H}(\omega, \theta) e^{-i \varphi_{A S H}(\omega, \theta)} u_{D}^{A}
\end{gathered}
$$

where $u_{N}^{S}, u_{N}^{A}$, and $u_{N}^{S H}$ represent the damage scattered S0, A0, and SH0 wave sources respectively. It should be stated clearly that, in this study, we focused our attention on fundamental Lamb modes ( $\mathrm{S} 0$ and $\mathrm{A} 0$ ) and fundamental shear horizontal mode (SH0), as corresponding to guided wave propagation in thin-wall structures below the A1 and SH1 critical frequency. The extension of our approach to higher modes will be done in a future study. These scattered waves are transferred from damage up to the sensing point. The 2-D Lamb wave field eradiating from a point source takes the following solution in the cylindrical coordinate system with respect to the damage location $[20,1]$ :

$$
u_{r}=\sum_{n=1}^{\infty} a_{n}^{\text {Lamb }}(z) H_{1}^{(1)}\left(\xi_{n}^{\text {Lamb }} r\right) e^{-i \omega t} ; \quad u_{\theta}=\sum_{n=1}^{\infty} a_{n}^{S H}(z) H_{0}^{(1)}\left(\xi_{n}^{S H} r\right) e^{-i \omega t}
$$

where $a_{n}^{L a m b}(z)$ and $a_{n}^{S H}(z)$ are the thickness dependent mode shapes for Lamb waves and shear horizontal waves of wave mode number $n$. Since the amplitude relationship between the incident waves and the scattered waves is enclosed in the WDICs, the transfer function from the damage up to the sensing point are simply $H_{1}^{(1)}\left(\xi R_{S C}\right)$ for Lamb waves and $H_{0}^{(1)}\left(\xi R_{S C}\right)$ for shear horizontal waves. $R_{S C}$ is the distance from the damage up to the sensing location. Thus, the scattered S0, A0, and SH0 waves arriving at the sensing point can be calculated.

$$
u_{S C}^{S}=u_{N}^{S} H_{1}^{(1)}\left(\xi^{S} R_{S C}\right) ; \quad u_{S C}^{A}=u_{N}^{A} H_{1}^{(1)}\left(\xi^{A} R_{S C}\right) ; \quad u_{S C}^{S H}=u_{N}^{S H} H_{0}^{(1)}\left(\xi^{S H} R_{S C}\right)
$$

STEP 5: The total wave field at the sensing location is the superposition of the direct incident 
waves calculated from Eq. (9) and the scattered waves calculated from Eq. (15).

$$
u_{T O T A L}=\left(u_{I N}^{S}+u_{I N}^{A}\right)+\left(u_{S C}^{S}+u_{S C}^{A}+u_{S C}^{S H}\right)
$$

It should be noted that the total wave field obtained in Eq. (16) is the in-plane wave displacement. Since SLDV measures out-of-plane particle velocity, we need to convert this in-plane wave motion into out-of-plane wave motion.

STEP 6: The out-of-plane displacement wave field can be obtained by the modeshape component ratio.

$$
u_{z}^{S}=u_{r}^{S} \frac{U_{z}^{S}(\omega, d)}{U_{r}^{S}(\omega, d)} ; \quad u_{z}^{A}=u_{r}^{A} \frac{U_{z}^{A}(\omega, d)}{U_{r}^{A}(\omega, d)} ; \quad u_{z}^{S H}=u_{\theta}^{S H} \frac{U_{z}^{S H}(\omega, d)}{U_{\theta}^{S H}(\omega, d)}
$$

where $U_{r}^{S}, U_{z}^{S}, U_{r}^{A}, U_{z}^{A}, U_{\theta}^{S H}, U_{z}^{S H}$ are Lamb wave and shear horizontal modeshape displacement components evaluated at the top surface of the structure. The modeshape solutions can be found in Ref. [1]. The out-of-plane velocity was calculated by differentiating the out-of-plane displacement with respect to time. Through this differentiation, the wave amplitude will be modified by a factor of $-i \omega$ as shown in Eq. (18).

$$
U=u \cdot e^{-i \omega t} ; \quad \frac{\partial U}{\partial t}=\frac{\partial\left(u \cdot e^{-i \omega t}\right)}{\partial t}=-i \omega \cdot U
$$

Thus, the solution for out-of-plane velocity in frequency domain can be obtained in the following form.

$$
\dot{u}_{z}\left(\omega, R_{D}, R_{S C}, \theta\right)=-i \omega \cdot u_{z}
$$

STEP 7: Perform inverse Fourier transform to obtain the time domain sensing signal.

$$
\dot{u}_{z}\left(t, R_{D}, R_{S C}, \theta\right)=\operatorname{IFFT}\left[\dot{u}_{z}\left(\omega, R_{D}, R_{S C}, \theta\right)\right]
$$

In this study, we focused our attention on fundamental modes of Lamb modes (S0 and A0) and fundamental shear horizontal mode (SH0), which find the widest application in Lamb wave based SHM. However, higher orders of Lamb modes (S1, A1, S2, A2, etc.) as well as shear horizontal (SH1, SH2, etc.) modes may also result from the wave-damage interaction if the damage has an arbitrary non-symmetric shape. The local FEM technique developed in this study were used to project the FEM response onto the S0, A0, SH0 modes and thus determine the WDICs resulting from the interaction between incident $\mathrm{S} 0$ or A0 modes with the specific damage. (It should be noted that we did not include the case of an incident SHO wave mode in our 3-D local FEM analysis because a circular PWAS transmitter cannot generate SH waves. However, scatter SH waves may result from the damage interaction process through the mode 
conversion). It should also be pointed out that, for model validation purpose, we used SLDV which measures the out-of-plane velocity of the wave field. Thus, the scattered SH waves, which do not have out-of-plane motion, cannot be captured.

\section{Analytical Predictive Tool Development: WaveFormReVealer 2-D}

The analytical model is a general description of wave generation, propagation, damage interaction, and detection. Parameter exploration is made possible for transmitter PWAS size, structure material and thickness, sensor-damage locations, and arbitrary excitations, etc. This analytical framework was coded using MATLAB, and a graphical user interface (GUI) called WaveFormRevealer 2-D (WFR-2D) was developed.

Figure 2a shows the WFR-2D main interface which calculates the real time sensing signals as well as dispersion curves and tuning curves. The parameter control panel allows users to modify host structure material properties, thickness, and transmitter-damage-sensing locations. The excitation control panel provides excitation waveform, frequency, and arbitrary excitation loading options. Users can also selectively choose the excited wave mode of interest. Figure $2 \mathrm{~b}$ shows the damage information platform for inputting wave-damage interaction coefficients (WDICs). Figure $2 \mathrm{c}$ and Figure $2 \mathrm{~d}$ show the sub-interfaces for loading S0 and A0 WDICs. The PWAS properties module allows users to define PWAS geometric and material properties (Figure 2e). The spatial propagation solver, shown in Figure $2 \mathrm{f}$, is like a $\mathrm{C}$-scan, which calculates the transient time-space domain wave field.
(a) WFR Main Interface
(b) Scatter Information Platform
(c) S0 WDICs Module

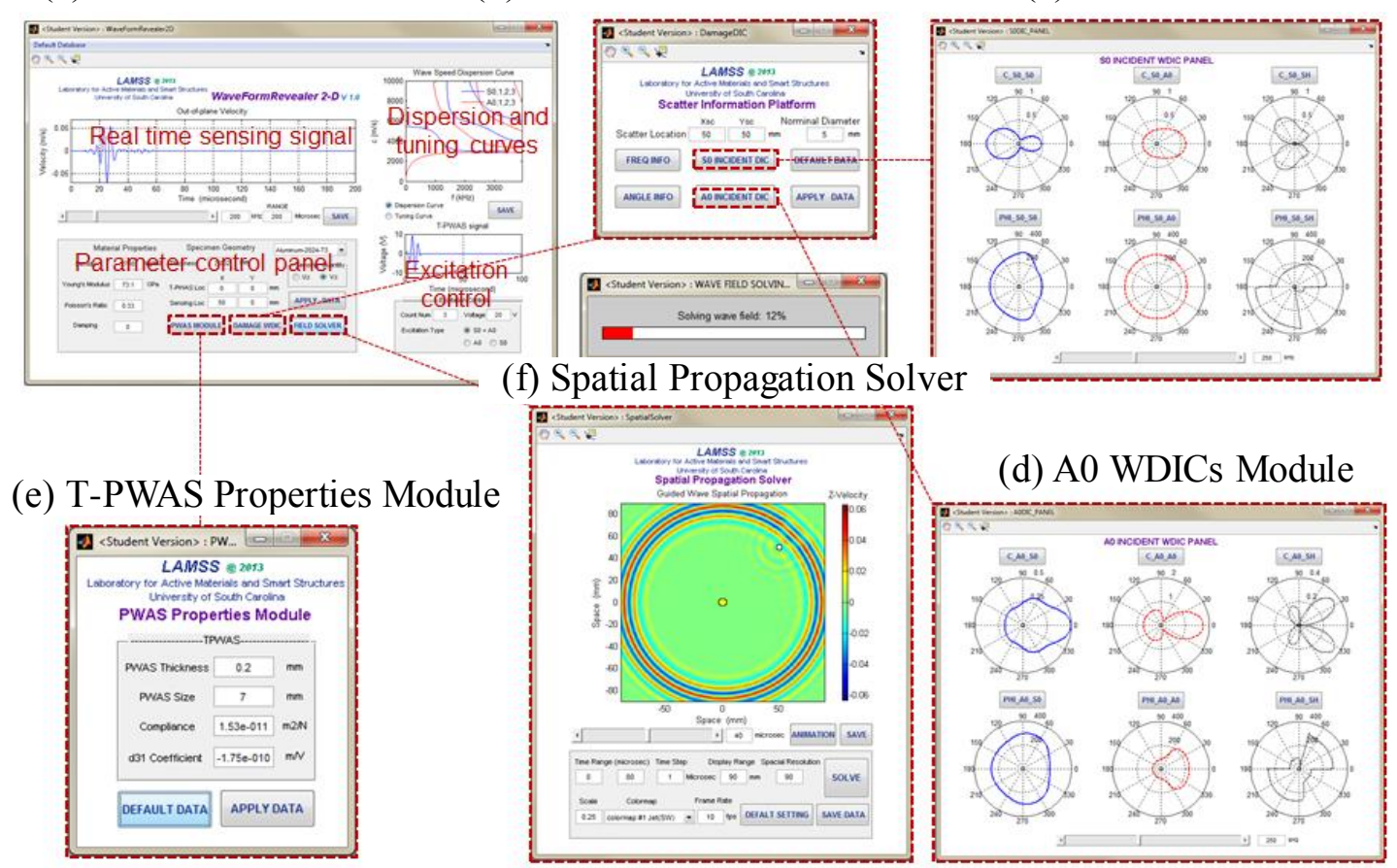

Figure 2: GUI of WFR-2D: (a) WFR-2D main interface; (b) damage information platform; (c) S0 WDICs module; (d) A0 WDICs module; (e) T-PWAS properties module; (f) spatial propagation solver. 
Users can visualize wave propagation and interaction with damage easily with the animation functions offered by WFR. Additionally, all the calculation data is available for user analysis with the data saving functions. The WFR approach is fast and efficient and can be used for SHM system design studies. Commercial FEM software codes may take tens of hours to predict accurately the ultrasonic 3-D wave-propagation response at practical locations away from the source. The WFR approach does this much faster: two or three hours for local FEM scatter calculation and only a few seconds for propagating the response from the scatter to the receiver PWAS. With this analytical tool, an increase in computational efficiency can be achieved for exploring a large number of SHM design parameters.

\section{EXTRACTION OF WDIC FROM LOCAL FEM}

The extraction of WDICs is the key for CAFA. To obtain these frequency and direction dependent complex-valued coefficients, harmonic analysis of local FEM were carried out.

\subsection{SMALL-SIZE LOCAL FEM}

In this study, commercial finite element package ANSYS 14 was used to implement and realize the local FEM model. Non-reflective boundaries (NRB) are used in the FEM harmonic analysis to simulate continuous harmonic waves which are incident to a region of interest, as well as waves scattering from an arbitrary damage [33, 34, 35, 36, 41]. This analysis can be performed for any specific frequency of interest. The steady-state amplitude and phase information facilitate the extraction of WDICs. It should be pointed out that the model described in Ref [33] allows the extraction of all scattering information from only one FE model. In this research, however, we tailored the local FEM procedure to better integrate with our global analytical framework. And to obtain the relative relationship of amplitude and phase between a certain incident wave mode and the scattered waves at the damage location, we constructed two models with one pristine and one damaged to extract the scattering features in a straight forward manner. It is also important to state clearly that this study only focuses on PWAS-generated fundamental S0 and A0 modes as incident waves interacting with the damage. Therefore, two pairs of (a total of four) harmonic analyses need to be performed: one pair for S0 incident waves and the other for A0 incident waves. In this 3D FEM model, SH0 mode is considered as a scattered wave from mode conversion, but not as an incident wave mode. Figure 3 shows the small-size FEM pair designed for a 2.032-mm thick 2024-T3 aluminum plate. Each model is $100 \mathrm{~mm}$ long, $100 \mathrm{~mm}$ wide, and $2.032 \mathrm{~mm}$ thick, with a $30 \mathrm{~mm}$ wide NRB covering each boundary.

Figure 3 also shows the loading nodes and the sensing nodes. The loading nodes are aligned to create a straight crested harmonic incident wave field, which is a good approximation when the damage is located far away from the excitation source. In the pristine model, a circle of sensing nodes and one center sensing point are designed to collect the structural harmonic response. The center sensing point records the incident waves arriving at the damage location. In the damaged case, only a circle of sensing nodes, with the same location as the pristine model, are 
used to collect the data. The design of the circular positioned sensing nodes allows us to extract information for all the directions. The benefit of a harmonic analysis lies in the fact that it can provide the structural response under all the frequencies of interest with only one execution of the simulation, i.e., in commercial FE software packages, one only needs to define the specific frequency range and step size, and the software will calculate the results for all the specified frequencies.

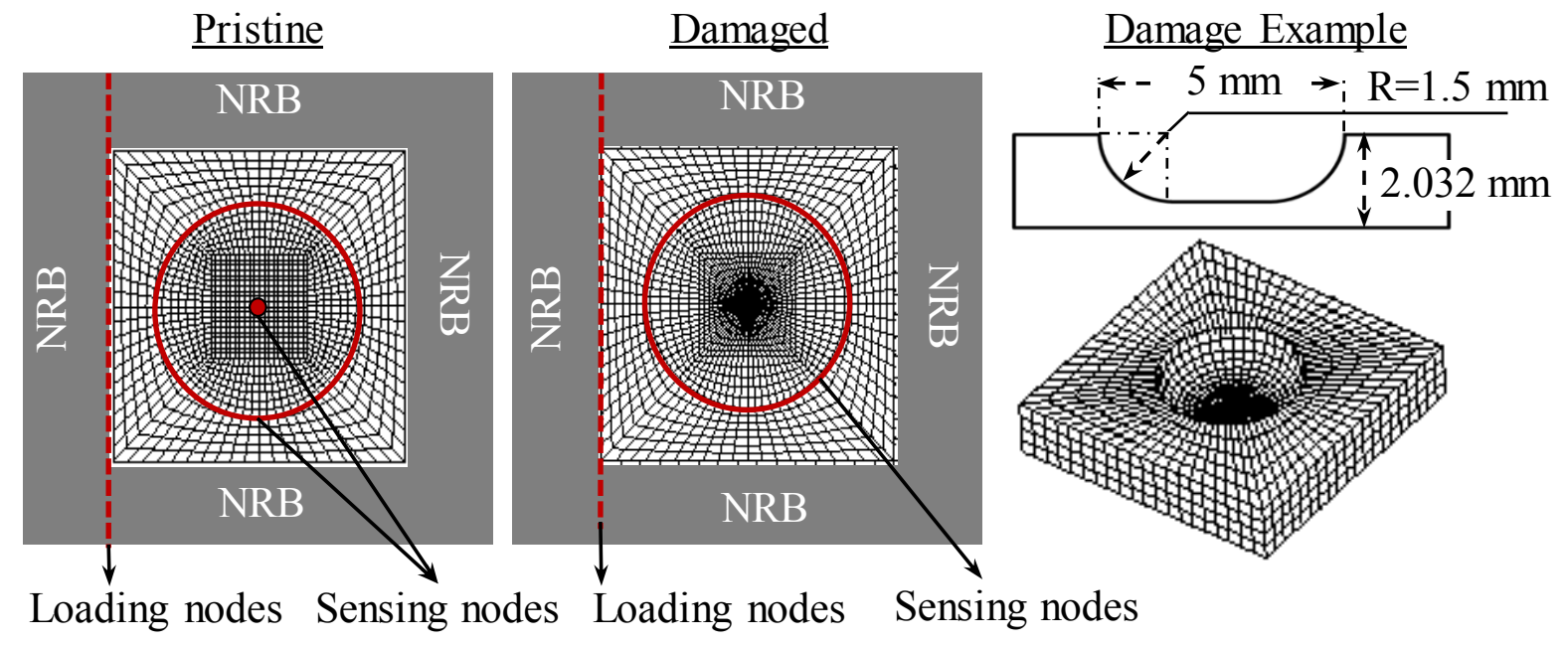

Figure 3: Small-size local FEM pair for WDICs extraction.

A damage example is shown in Figure 3. The geometry of damage is kept simple to ensure consistent FEM and experimental models, but complex enough to represent general wave-damage interaction phenomena, which have many mode conversion possibilities in the given frequency range. It should be noted that different types of damage will have different scattering characteristics; this will require a corresponding local damage model for WDIC extraction. It should also be noted that the harmonic analysis can only compute linear interaction between Lamb waves and damage.

\subsection{WDIC EXTRACTION PROCEDURE}

Figure 4 shows the schematic of the sensing region from the local FEM for the extraction of WDICs. The in-plane displacements at the sensing nodes are used. According to Eq. (2), the scattered wave field can be obtained by the subtraction of the incident waves from the total waves.

$$
W_{S C}=W_{\text {TOTAL }}-W_{I N}
$$

The sensing nodes data in the pristine model represent the incident wave field, while the data from damaged model correspond to the total wave field containing both incident and scattered waves. Thus, the subtraction of the data between these two models provides the scattered wave field in all directions. Using Eq. (21) and a transformation from Cartesian to polar coordinate system, we calculated the scattered wave displacements at the top and bottom surface sensing 
nodes in both radial $\left(u_{r}^{T}\right.$ and $\left.u_{r}^{B}\right)$ and tangential $\left(u_{\theta}^{T}\right.$ and $\left.u_{\theta}^{B}\right)$ directions. Using Eq. (22), we can separate the contribution from each scattered wave mode. It should be noted that pioneer research on local FEM techniques enables the extraction of scattering information of any Lamb modes from the damage, i.e., this technique is not limited to fundamental modes [33, 36]. The WIDC extraction procedure involving higher wave modes would require the solution throughout the thickness of the sensing boundary, instead of top and bottom surface only. By solving an overdetermined system of equations utilizing the solutions across the thickness, one can determine the participation of each wave mode. However, it is critical to state clearly that the extraction methodology considered in current study was designed especially for only fundamental plate guided wave modes (S0, A0, and SH0). The CAFA approach for higher wave modes should be investigated in a future study.

$$
u_{S C}^{S 0}=\frac{u_{r}^{T}+u_{r}^{B}}{2} ; \quad u_{S C}^{A 0}=\frac{u_{r}^{T}-u_{r}^{B}}{2} ; \quad u_{S C}^{S H 0}=\frac{u_{\theta}^{T}+u_{\theta}^{B}}{2}
$$

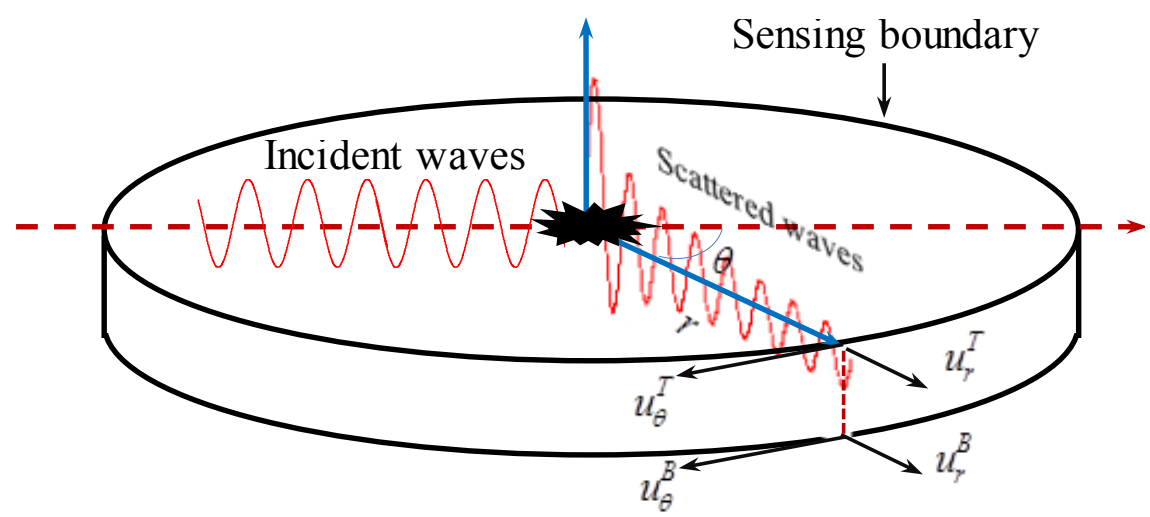

Figure 4: Extraction of WDICs from small-size FEM.

The incident waves arriving at the damage location are recorded by the center sensing point (in pristine local model) and denoted as $u_{I N}$. The relationship between the incident waves arriving at the damage and the scattered waves picked up on the sensing boundary can be formulated as Eq. (23).

$$
u_{I N}^{\mathrm{A}} e^{-i \varphi_{I N}^{\mathrm{A}}} \cdot C_{\mathrm{AB}}(\omega, \theta) e^{-i \varphi_{\mathrm{AB}}(\omega, \theta)} \cdot H_{m}^{(1)}\left(\xi^{\mathrm{B}} r\right)=u_{S C}^{\mathrm{B}}(\theta) e^{-i \varphi_{S C}^{\mathrm{B}}(\theta)}
$$

where $u_{I N}^{\mathrm{A}} e^{-i \varphi_{I N}^{\mathrm{A}}}$ is the $A$ mode incident wave recorded by the center sensing node with amplitude and phase information; $C_{\mathrm{AB}}(\omega, \theta) e^{-i \varphi_{\mathrm{AB}}(\omega, \theta)}$ is the WDIC, containing mode conversion ( $A$ mode to $B$ mode), direction dependency, amplitude ratio, and phase relationship 
information; $H_{m}^{(1)}\left(\xi^{\mathrm{B}} r\right)$ represents the outward propagating 2-D wave field of the resulting scattered wave mode $B$, with $m=1$ for Lamb waves and $m=0$ for SH waves. $u_{S C}^{\mathrm{B}}(\theta) e^{-i \varphi_{S C}^{\mathrm{B}}(\theta)}$ is the resulting scattered waves recorded on the sensing boundary with scatter angle, amplitude and phase information.

The harmonic analysis of the local FEM can provide the incident and scattered wave amplitude-phase information in Eq. (23), with $C_{\mathrm{AB}}(\omega, \theta) e^{-i \varphi_{\mathrm{AB}}(\omega, \theta)}$ left as the only unknown term. Upon rearrangement, Eq. (23) becomes

$$
C_{\mathrm{AB}}(\omega, \theta) e^{-i \varphi_{\mathrm{AB}}(\omega, \theta)}=\frac{u_{S C}^{\mathrm{B}}(\theta)}{u_{I N}^{\mathrm{A}}} \frac{1}{H_{m}^{(1)}\left(\xi^{\mathrm{B}} r\right)} e^{-\Delta \varphi_{\mathrm{AB}}(\theta)} ; \quad \Delta \varphi_{\mathrm{AB}}(\theta)=\varphi_{S C}^{\mathrm{B}}(\theta)-\varphi_{I N}^{\mathrm{A}}
$$

By identification, the amplitude coefficient and phase coefficient can be extracted.

$$
\begin{gathered}
C_{\mathrm{AB}}(\omega, \theta)=\left|\frac{u_{S C}^{\mathrm{B}}(\theta)}{u_{I N}^{\mathrm{A}}} \frac{1}{H_{m}^{(1)}\left(\xi^{\mathrm{B}} r\right)}\right| \\
\varphi_{\mathrm{AB}}(\omega, \theta)=\Delta \varphi_{\mathrm{AB}}(\theta)-\left[\angle \frac{1}{H_{m}^{(1)}\left(\xi^{\mathrm{B}} r\right)}-\angle \frac{1}{H_{m}^{(1)}\left(0^{+}\right)}\right]
\end{gathered}
$$

\subsection{EXAMPLES OF WDICS}

Figure 5 shows an example of S0 wave interaction with damage at $200 \mathrm{kHz}$. The first row shows the amplitude coefficients and the second row shows the phase coefficients. It can be observed that the interaction between the incident S0 wave and the damage not only involves scattered S0 wave, but also involves mode converted A0 and SH0 waves. Also, the amplitude and phase coefficients are heavily direction-dependent. The calculation of amplitude coefficients has been investigated in many literatures with various methods, but the estimation of phase coefficients only appeared in a few publications. For example, the model in Ref [33] allows the extraction complex valued scattering coefficients, which have been used to simulate ultrasonic array data or to solve inverse scattering problems [31, 37]. In this study, we obtained the phase information of the scattered waves to solve a forward scattering problem. The phase coefficients are of great significance in simulating wave-damage interaction, because they govern whether the incident and scattered waves will superpose constructively or destructive with each other. Figure 6 shows the WDICs for the incident A0 wave at $200 \mathrm{kHz}$. Similar scattering, mode conversion and directivity phenomena can be noticed in the coefficient patterns. 


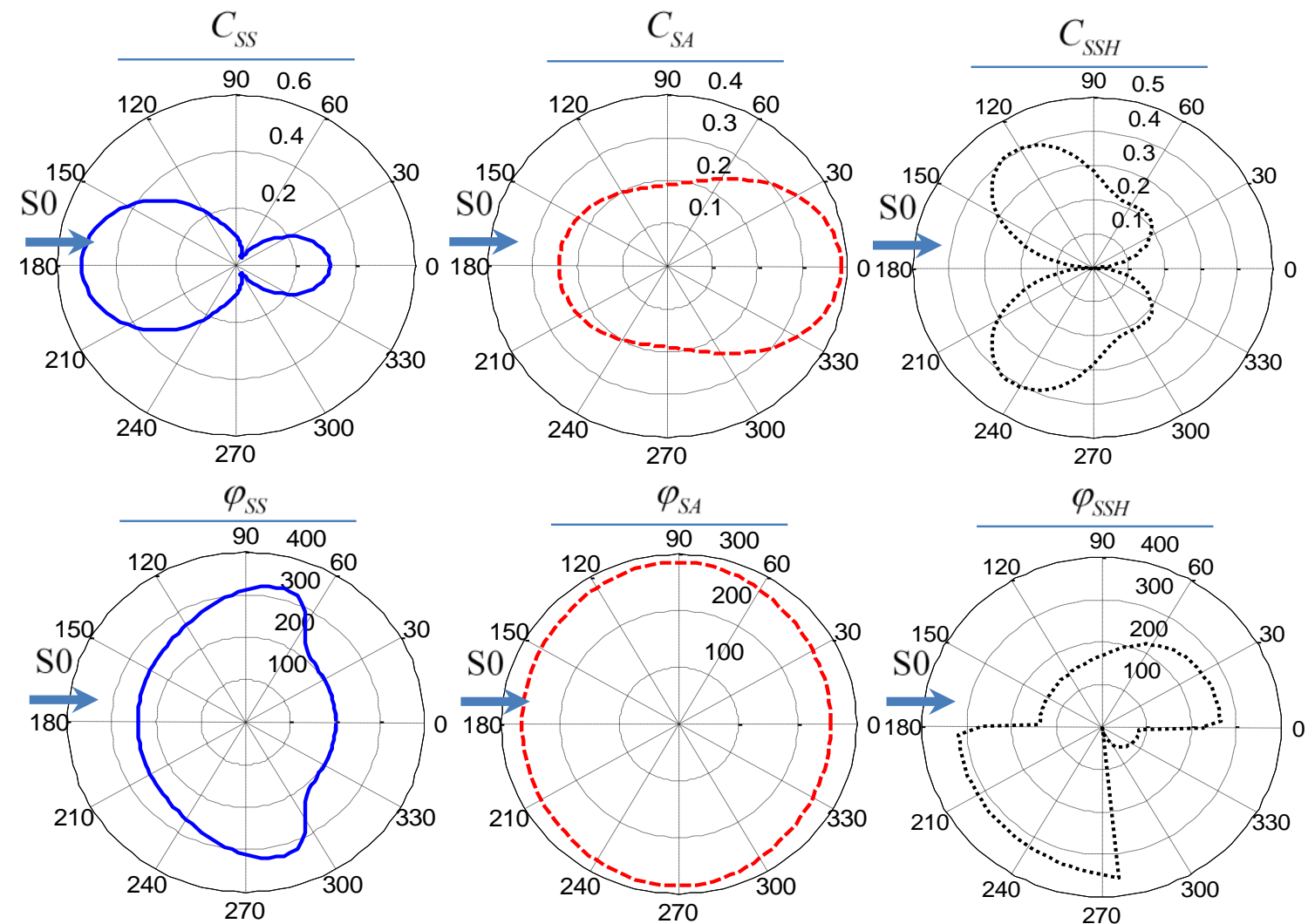

Figure 5: WDICs example of S0 wave interaction with damage at $200 \mathrm{kHz}$

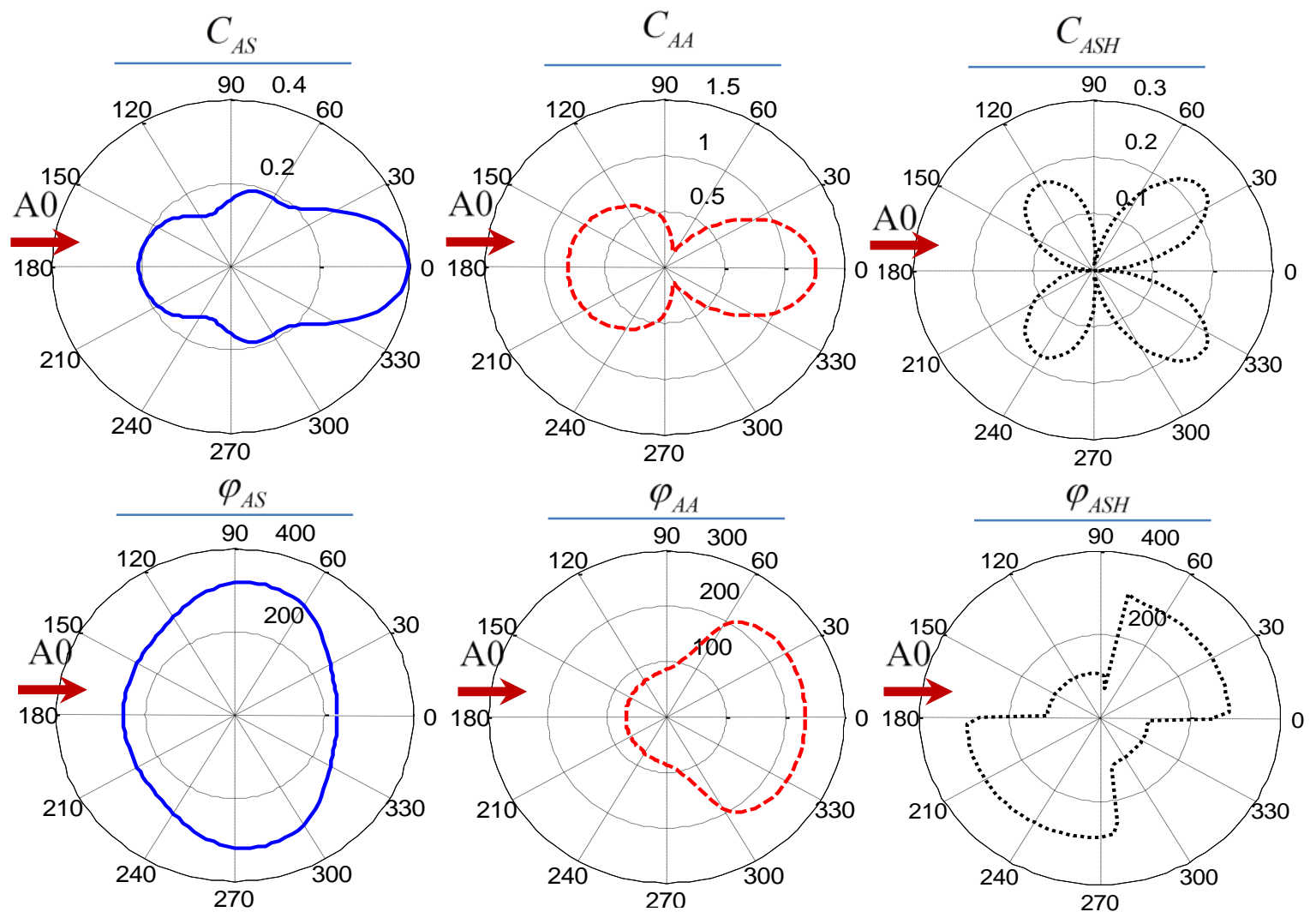

Figure 6: WDICs example of A0 wave interaction with damage at $200 \mathrm{kHz}$. 


\section{FULL-SCALE FEM AND EXPERIMENTAL VERIFICATIONS}

In this section, we will present the multiphysics full-scale FEM simulation and experiments with SLDV for CAFA verification. Case studies were carried out for Lamb wave propagation in a pristine plate and a damaged plate.

\subsection{MULTI-PHYSICS FULL-SCALE FEM SIMULATION}

Figure 7 shows the full-scale multi-physics finite element model constructed with the commercial FEM package ANSYS 14. The specimen is a $2.032 \mathrm{~mm}$ thick aluminum plate. To minimize the calculation burden, NRB were implemented around the model. We used SOLID5 coupled field elements to simulate the piezoelectric effect of T-PWAS, SOLID45 eight node structure element to mesh the plate, and COMBIN14 spring-damper element to construct the NRB. The mesh size adopted in this study is $1 \mathrm{~mm}$ for in-plane direction and $0.5 \mathrm{~mm}$ for thickness direction. The T-PWAS and damage regions were meshed with even smaller elements to accommodate the high stress gradient. A total of 423,468 elements were used. The time step was set to $0.25 \mu$ s. Two sets of simulations were carried out: (1) 2-D Lamb wave propagation in a pristine plate; (2) 2-D Lamb wave propagation in a damaged plate. The location and geometry information of the T-PWAS, damage, and specimen are shown in Figure 7.

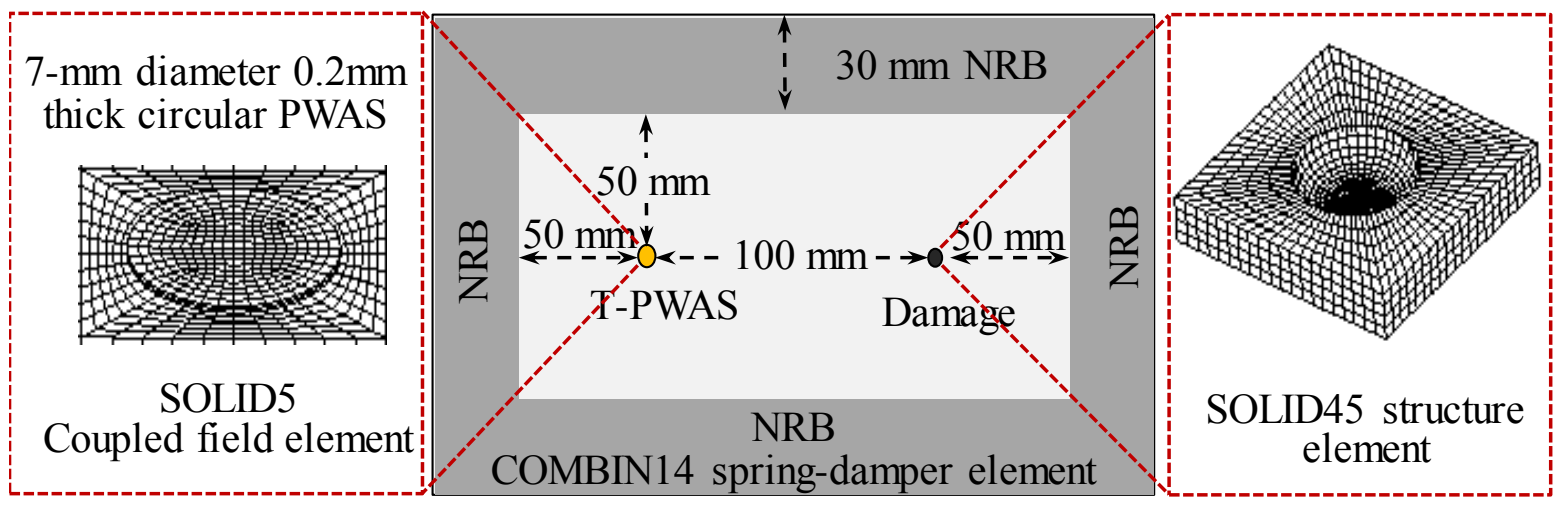

Figure 7:Multi-physics full-scale finite element model.

\subsection{EXPERIMENTS WITH SLDV}

Figure 8 shows the SLDV experimental setup. An Agillent 33522B function generator was used to generate a 3-count Hanning window modulated tone burst which was amplified to 50 Vpp through a Krohn-Hite 7600 wideband power amplifier and then applied to the T-PWAS. (The Krohn-Hite 7600 has a $200 \mathrm{Vpp}$ range from DC to $1 \mathrm{MHz}$, hence a linear amplification of the excitation signal without modifying its frequency content is expected). Lamb waves generated by the T-PWAS propagated along the structure, interacted with the damage, and were measured by a Polytec PSV-400 scanning laser Doppler vibrometer. The quantity measured by the SLDV is the out-of-plane velocity of surface particle motion. Reflective tapes were used to 
enhance the surface reflections and improve visualization quality. C-scans of the specimen surface were carried out for both the pristine and the damaged plate. The locations of the T-PWAS, damage, and special recording points are illustrated.

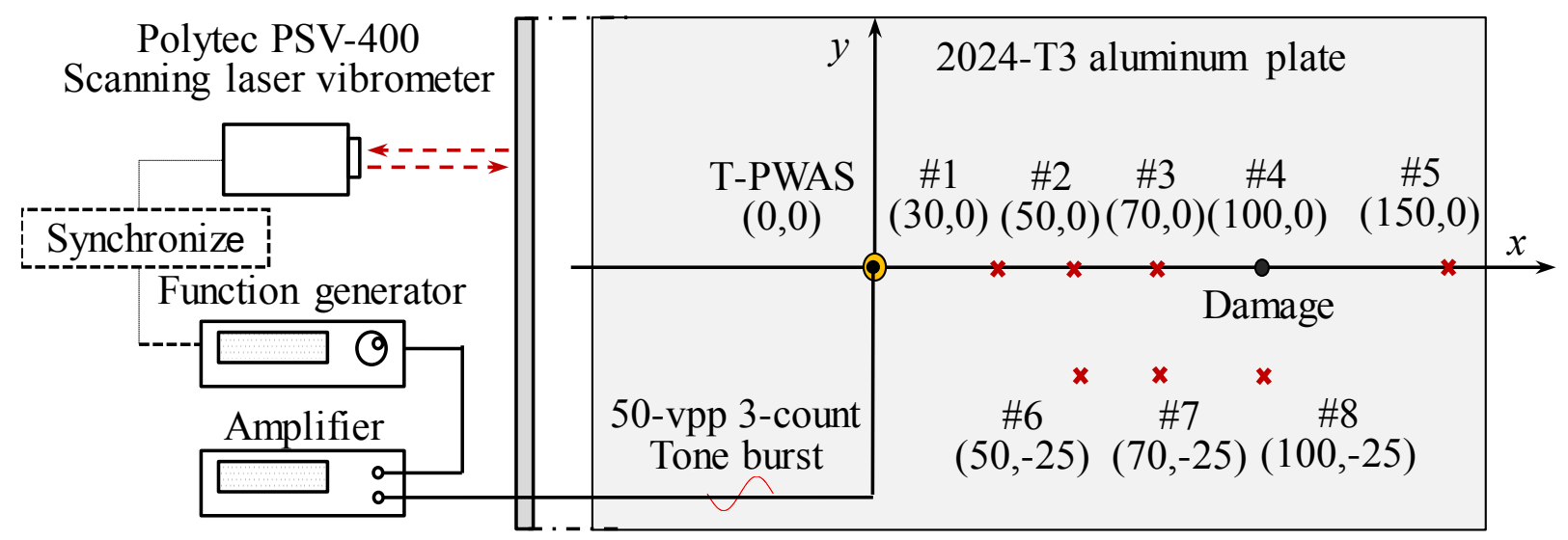

Figure 8: Experiment setup with SLDV.

\section{RESULTS AND DisCUSSION}

\subsection{Wave Propagation in a Pristine Plate}

Figure 9 shows the comparison of the transient spatial wave field among CAFA, full-scale FEM simulation, and SLDV measurement in a pristine plate. It can be observed that CAFA predictions have good agreement with both full-scale FEM simulation and SLDV experiment. Lamb waves were generated by the T-PWAS, propagating out in a circular wave front, strong near the wave source, and weak at far field due to the outward propagation pattern.

(a)

CAFA prediction $10 \mu$
Lamb waves being
generated by T-PWÂS
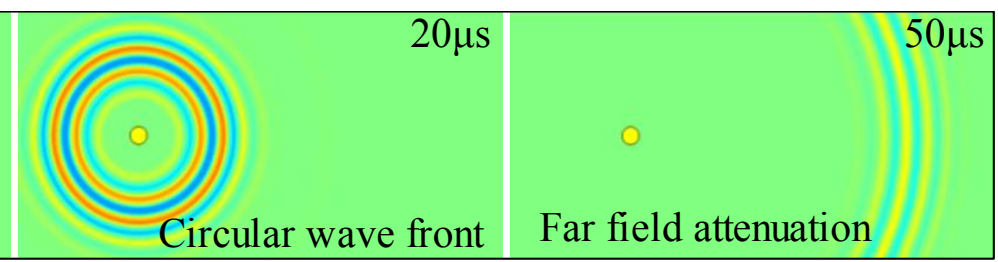

(b)

(C)

(c)

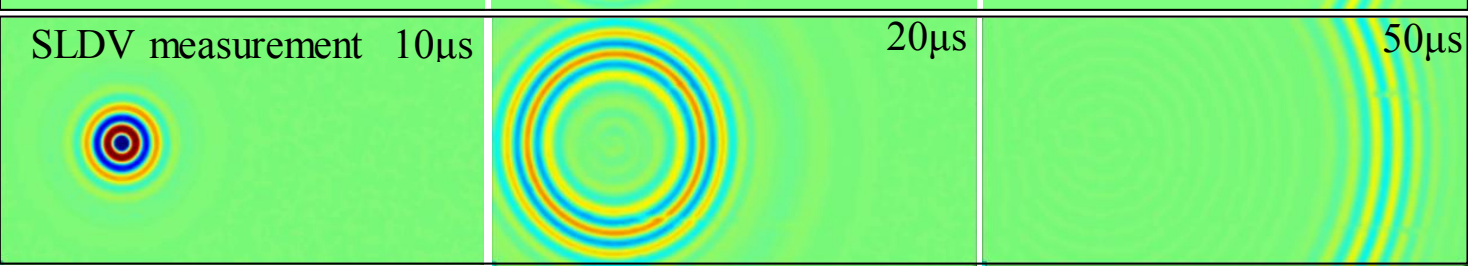

Figure 9: Comparison of $200 \mathrm{kHz}$ wave field in the pristine plate: (a) CAFA prediction; (b) full-scale FEM simulation; (3) experiment with SLDV. 
Figure 10 shows the waveform validation results at various sensing locations at $200 \mathrm{kHz}$. It can be observed that CAFA predictions have good agreement with both FEM simulation and experiments. At the near field (location \#2), S0 and A0 waves are mixed together. The FEM result shows a shift from the CAFA prediction, while CAFA prediction agrees well with the laser measurement. The FEM mesh adopted in this study does not capture the near field waves as accurate as CAFA. Of course, we can improve the accuracy of FEM simulation by further discretizing the analyzed domain and adopting smaller time step, but such improvement in accuracy requires additional computational resources and a much longer calculation time. For higher frequency simulations, full scale 3-D FEM becomes prohibitive. It can also be observed that CAFA prediction of the far field waveform agrees well with both full-scale FEM simulation and SLDV experiments for a pristine plate.

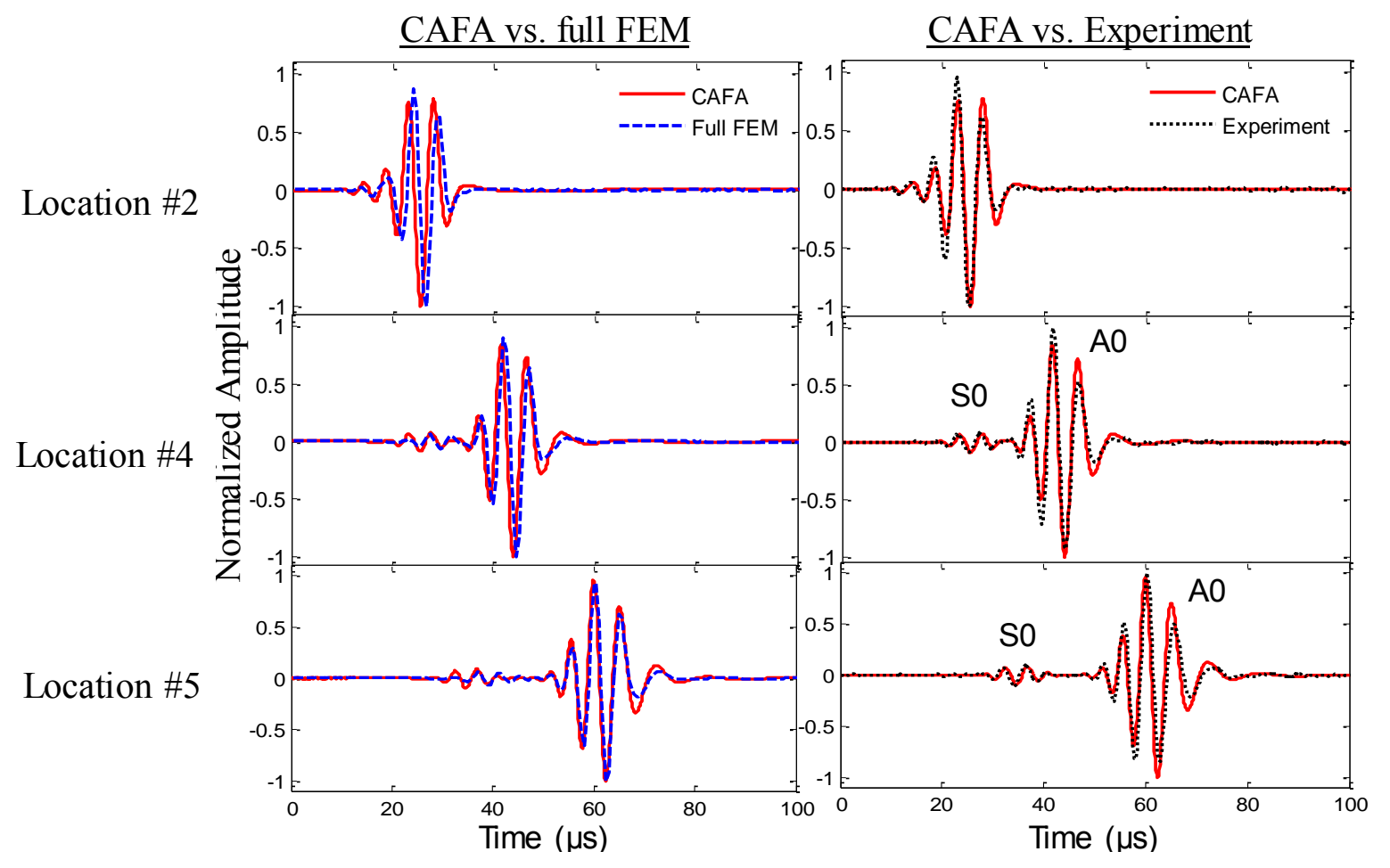

Figure 10: Results comparison of $200 \mathrm{kHz}$ signals of pristine plate at various sensing locations shown in Figure 8.

\subsection{WaVe Propagation in a Damaged Plate}

Figure 11 shows the comparison of a transient spatial wave field among CAFA, full-scale FEM simulation and SLDV measurement in a damaged plate. It can be observed that the CAFA results agree well with full-scale FEM simulation and experimental measurement. At $30 \mu \mathrm{s}$, the fast propagating S0 mode with a long wavelength and the slowly propagating A0 mode with short wavelength can be clearly identified. The mode converted A0 waves can be noticed, propagating with a short wavelength from S0 interaction with the damage. At $50 \mu$ s, after A0 waves interact with the damage, the scattered A0 mode can be observed as well as the shadings 
left behind the damage. These shadings are caused by the destructive superposition between the incident A0 waves and the scattered A0 waves. This also illustrates the importance of obtaining phase information in the WDICs. It should be noted that measurement discontinuities were found in the experimental data, which were caused by the reflective tape boundaries. It should also be pointed out that, in order to compare with the SLDV measurement, we are comparing the out-of-plane velocity of the wave fields. Thus, the SH modes which do not have out-of-plane components (from their mode shapes) will not present in the out-of-plane field plots.
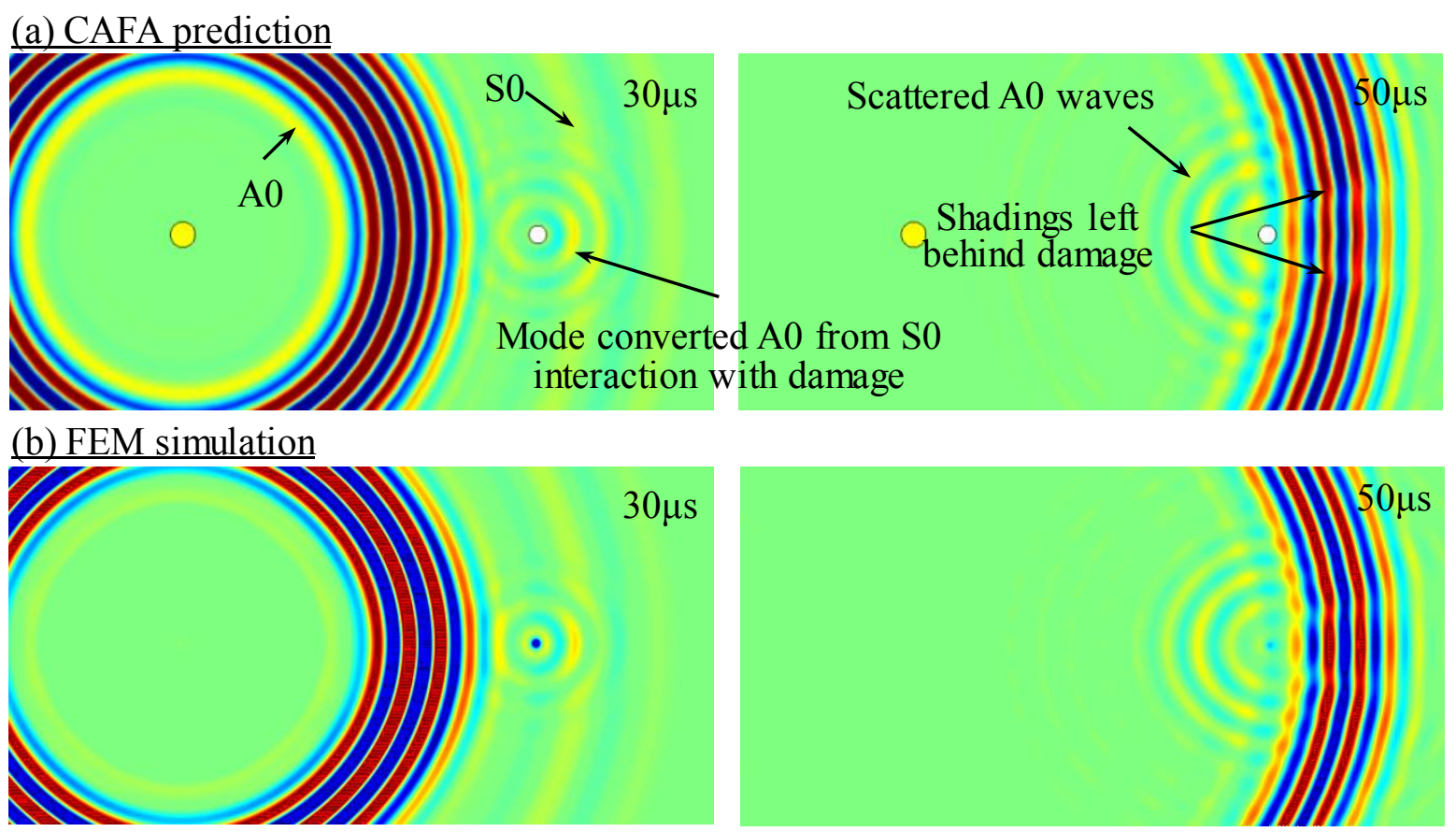

\section{(c) SLDV measurement}

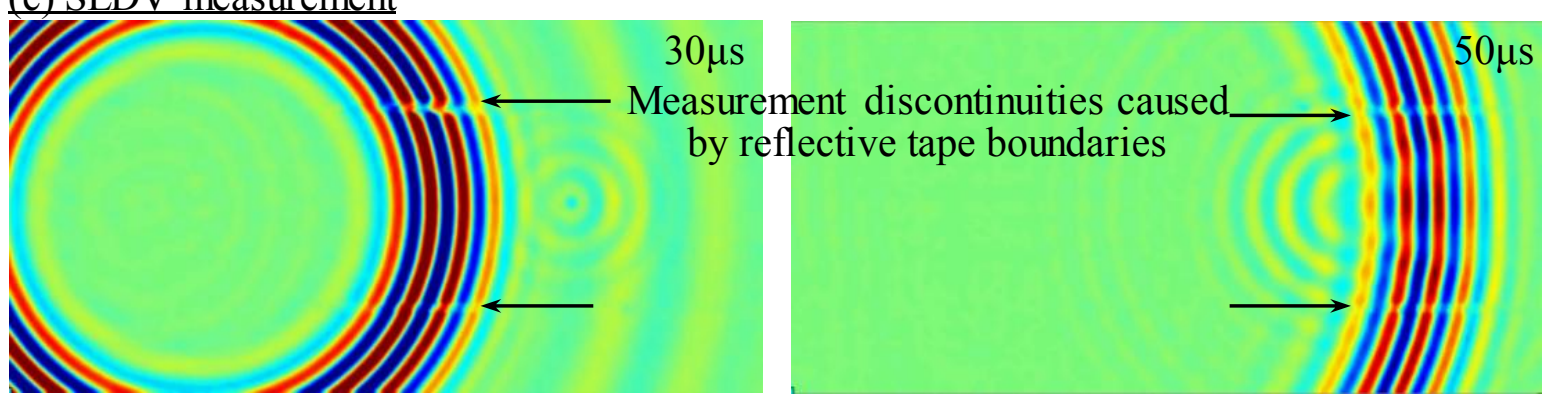

Figure 11: Comparison of wave field in a damaged plate, showing S0 and A0 Lamb modes interacting with the damage: (a) CAFA prediction; (b) full-scale FEM simulation; (c) SLDV experiment.

Figure 12 shows the waveform validation results at various sensing locations for a $200 \mathrm{kHz}$ excitation. It can be observed that WFR predictions agree well with full-scale FEM simulation and experiments. The signals at location \#1, \#2, and \#3 show that the scattered A0 wave amplitude increases when the sensing location moves closer to the damage. The signal 
at location \#5 shows the mode converted A0 wave packet from the S0 interaction with damage.

Figure 13 shows the comparison of the sensing signals at locations \#6, \#7, and \#8, which are in certain directions with respect to the incident waves and the damage. It should be noted that at location \# 6 and \#7, damage scattered waves are clearly observed, but at location \#8, no damage effect can be seen. Recall the WDICs pattern in Figure 6, the scattered A0 amplitude coefficient reaches a minimum value near 90 degrees with respect to the incident waves. This fact illustrates that at certain sensing locations, the damage cannot be effectively detected. Using CAFA and WFR-2D, the waveforms can be predicted at arbitrary sensing locations, and the users can determine whether these locations are sensitive points or blind zones, optimizing the design of a sensor network for damage detection.

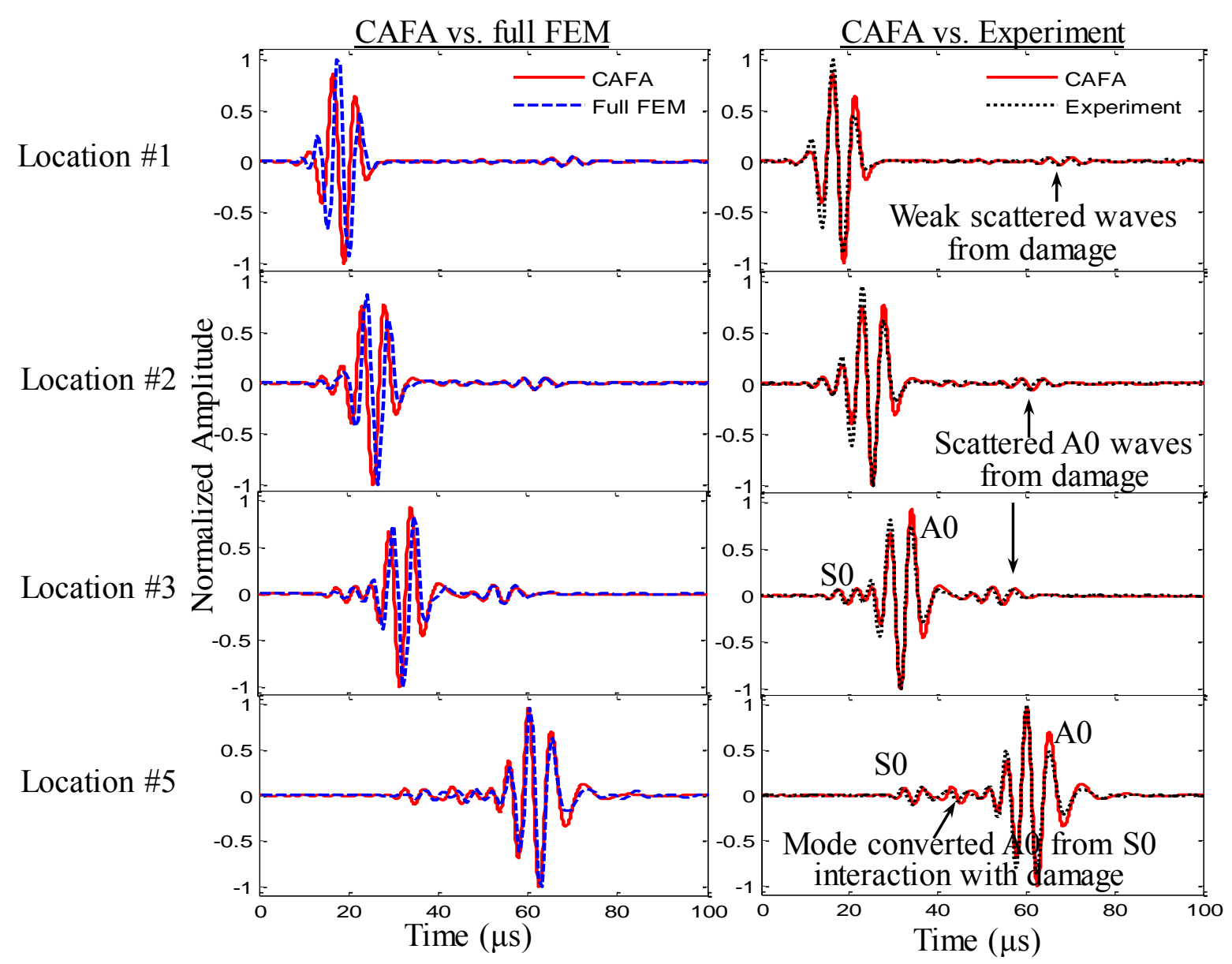

Figure 12: Results comparison of $200 \mathrm{kHz}$ signals of damaged plate at locations \#1 through \#5. 


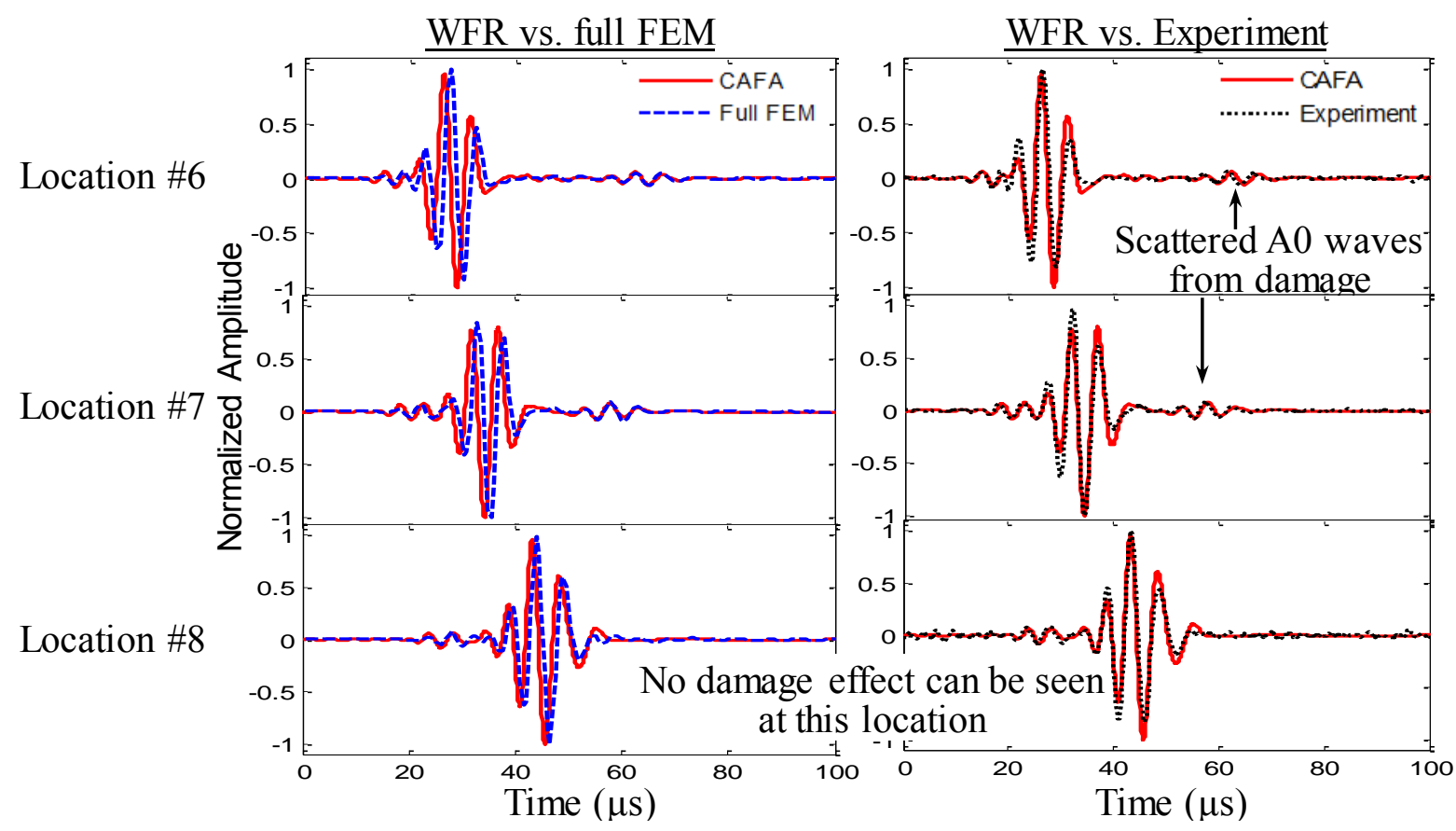

Figure 13: Results comparison of $200 \mathrm{KHz}$ signals of damaged plate at locations \#6 through \#8.

\subsection{AdVANTAGE OF CAFA OVER OTHER COMPUTATIONAL TECHNIQUES}

CAFA can deal with transient wave interaction with complicated damage type and geometries in an easier and more user-friendly way through WFR-2D, than other analytical or semi-analytical formulations such as distributed point source method (DPSM) and semi-analytical finite element (SAFE) method.

CAFA does not require meshing of the entire structure, thus it minimizes the computation burden; the analytical framework is constructed in the frequency domain, and does not require a time marching procedure, thus it saves considerable computation time, compared with conventional numerical methods such as finite element method (FEM), spectral element method (SEM), or finite difference method (LISA).

Because it is fast and efficient, CAFA can be used for parametric studies for a large family of SHM problems. It enables researchers to define transducer size, structure material and thickness, sensor-damage locations, and arbitrary excitations by interacting with the GUI of WFR-2D. This user-friendly feature and parameter definition freedom allows users to explore the best sensor arrangement for damage detection in a highly efficient manner, whereas any of these parameter changes will result in re-built/re-mesh/re-run of the whole model in the case of conventional FEM simulation.

Besides, the conventional FEM needs to save all the nodal solutions for each time marching step, which is a memory consuming procedure and requires large hard drive space for data storage. However, CAFA generates a 2-D wave field data with much less computer resource consumption. 
Taking the examples in this study, for each test frequency, it takes more than 20 hours for the full-scale FEM simulation. However, CAFA only consumes 2 to 3 hours local FEM computation and several minutes' analytical calculation (WFR-2D). Conventional FEM requires re-model and re-run for the exploration of design parameters such as PWAS transducer size, sensor-damage relative location, interrogating wave frequency, and excitation waveform; however, CAFA only requires the modification of the corresponding calculation parameters and the exploration for each parameter only takes several minutes. Thus, CAFA can achieve orders of magnitude higher design efficiency when compared with conventional FEM. The data size of conventional 3-D FEM simulation can reach several hundreds of Gigabytes, while the CAFA 2-D time space wave field only consumes several hundreds of Megabytes.

\section{CONCLUSIONS AND FUTURE WORK}

\subsection{CONCLUSIONS}

In this research, a methodology was proposed for inserting damage effects into the 2-D Lamb wave analytical model. CAFA was developed as a complete model which couples the analytical framework with local FEM for the simulation of 2-D Lamb wave damage detection. WFR-2D was developed as the user-friendly software to conduct fast predictive simulations and parametric studies. The CAFA results compared well with full-scale multi-physics FEM simulations and SLDV experiments. It was found that CAFA achieved remarkable performance in terms of computational accuracy, efficiency, and versatility.

\subsection{FUTURE WORK}

CAFA verification with other types of damage should be performed. Extension to more than the basic S0, A0, and SH0 scattering modes should be made. Inclusion of nonlinear effects in CAFA should be attempted. WFR concept should be developed to simulate wave propagation in composite structures. CAFA should be explored to solve wave propagation in structures with more complicated geometries and should include boundary reflections. The analytical framework should be further investigated to include thermal and loading effects to consider wave generation, propagation, damage interaction, and detection under various temperature and loading conditions.

\section{ACKNOWLEDGEMENTS}

Support from Office of Naval Research \# N00014-11-1-0271, Dr. Ignacio Perez, Technical Representative; Air Force Office of Scientific Research \#FA9550-11-1-0133, Dr. David Stargel, Program Manager; SPARC fellowship at University of South Carolina; are thankfully acknowledged. 


\section{REFERENCES}

[1] V. Giurgiutiu, Structural Healthing Monitoring with Piezoelectric Wafer Active Sensors (Second Edition), Elsevier Academic Press, 2014.

[2] A. Raghavan and C. Cesnik, "Finite-dimensional piezoelectric transducer modeling for guided wave based structural health monitoring," Smart Materials and Structures, vol. 14, pp. 1448-1461, 2005.

[3] Y. Shen and V. Giurgiutiu, "WaveFormRevealer: An Ananlytical Framework and Predictive Tool for the Simulation of Multi-modal Guided Wave Propagation and Interaction with Damage," Structural Health Monitoring -- An International Journal, p. DOI: 10.1177/1475921714532986, 2014.

[4] A. N. Norris and C. Vemula, "Scattering of flexual waves in thin plates," Journal of Sound and Vibration, pp. 115-125, 1995.

[5] C. Vemula and A. N. Norris, "Flexual wave propagation and scattering on thin plates using Mindlin theory," Wave Motion, vol. 26, pp. 1-12, 1997.

[6] C. Wang and F. Chang, "Scattering of plate waves by a cylindrical inhomogeneity," Journal of Sound and Vibration, vol. 282, pp. 429-451, 2005.

[7] L. Moreau, M. Caleap, A. Velichko and P. D. Wilcox, "Scattering of guided waves by through-thickness cavities with irregular shapes," Wave Motion, vol. 48, pp. 586-602, 2011.

[8] L. Moreau, M. Caleap, A. Velichko and P. D. Wilcox, "Scattering of guided waves by flat-bottomed cavities with irregular shapes," Wave Motion, p. doi: 10.1016/j.wavemoti.2011.12.004, 2012.

[9] J. C. P. McKeon and M. K. Hinders, "Lamb waves scattering from a through hole," Journal of Sound and Vibration, pp. 843-862, 1999.

[10] T. Grahn, "Lamb wave scattering from a circular partly through-thickness hole in a plate," Wave Motion, 2002.

[11] M. K. Hinders, "Lamb waves scattering from rivets," Quantitative Nondestructive Evaluation, vol. 15, 1996.

[12] D. Alleyne and P. Cawley, "The interaction fo Lamb waves with defects," IEEE Transactions on Ultrasonics Ferroelectrics and Frequency Control, vol. 39, no. 3, pp. 381-397, 1992.

[13] Y. Shen and V. Giurgiutiu, "Predictive modeling of nonlinear wave propagation for 
structural health monitoring with piezoelectric wafer active sensors," Journal of Intelligent Material Systems and Structures, vol. 25, no. 4, pp. 506-520, 2014.

[14] Y. Cho, D. Hongerholt and J. Rose, "Estimation of ultrasonic guided wave mode conversion in a plate with thickness variation," IEEE Transaction on Ultrasonics, Ferroelectrics, and Frequency Control, vol. 47, no. 3, 2000.

[15] J. Galan and R. Abascal, "Boundary element solution for the bidimensional scattering of guided waves in laminated plates," Computers \& Structures, vol. 83, pp. 740-757, 2005.

[16] W. Ostachowicz, P. Kudela, M. Krawczuk and A. Zak, Guided Waves in Structures for SHM: The Time-Domain Spectral Element Method, West Sussex, UK: Wiley, 2012.

[17] B. Lee and W. J. Staszewski, "Lamb wave propagation modelling for damage detection: I. Two-dimensional analysis," Smart Materials and Structures, pp. 249-259, 2007.

[18] K. Nadella and C. Cesnik, "Local interaction simulation approach for modeling wave propagation in composite structures," CEAS Aeronaut Journal, vol. 4, pp. 35-48, 2013.

[19] E. Rahani and T. Kundu, "Modeling of Transient Ultrasonic Wave Propagation Using the Distributed Point Source Method," IEEE Transactions on Ultrasonics, Ferroelectrics, and Frequency Control, vol. 58, no. 10, 2011.

[20] E. Glushkov, N. Glushkova, R. Lammering, A. Eremin and M. Neumann, "Lamb wave excitation and propagation in elastic plates with surface obstacles: proper choice of central frequency," Smart Materials and Structures, p. 11pp, 2011.

[21] A. Srivastava, Quantitative structural health monitoring using ultrasonic guided waves, PhD Thesis, University of California, San Diego, CA, 2009.

[22] F. Benmeddour, F. Treyssede and L. Laguerre, "Numerical modeling of guided wave interaction with non-axisymmetric cracks in elastic cylinders," International Journal of Solids and Structures, pp. 764-774, 2011.

[23] Z. Ahmad, J. Vivar-Perez and U. Gabbert, "Semi-analytical finite element method for modeling of Lamb wave propagation," CEAS Aeronaut Journal, vol. 4, pp. 21-33, 2013.

[24] D. Goetschel, S. Dong and B. Muki, "A global local finite element analysis of axisymmetric scattering of elastic waves," Journal of Applied Mechanics, pp. 816-820, 1982.

[25] Z. Chang and A. Mal, "Scattering of Lamb waves from a rivet hole with edge cracks," Mechanics of Materials, pp. 197-204, 1999.

[26] N. Terrien, D. Doyer, F. Lepoutre and A. Deom, "Numerical predictions and experiments for optimizing hidden corrosion detection in aircraft structures using Lamb modes," 
Ultrasonics, pp. 251-265, 2007.

[27] L. Moreau and M. Castaings, "The use of an orthogonality relation for reducing the size of fintie element models for 3D guided waves scattering problems," Ultrasonics, pp. 375-366, 2008.

[28] M. Gresil and V. Giurgiutiu, "Time-domain global-local concept for guided-wave propagation with piezoelectric wafer active sensor," Journal of Intelligent Material Systems and Structures, vol. 24, no. 15, pp. 1897-1911, 2013.

[29] M. Gresil and V. Giurgiutiu, "Time-domain global-local prediction of guided waves interaction with damage," Key Engineering Materials, vol. 559, pp. 116-127, 2013.

[30] M. Obenchain, K. Nadella and C. Cesnik, "Hybrid global matrix/local interaction simulation approach for wave propagation in composites," AIAA Journal, p. DOI: 10.2514/1.J053101, 2014.

[31] J. Zhang, B. Drinkwater, P. Wilcox and A. Hunter, "Defect detection using ultrasonic arrays: The multi-mode total focusing method," NDT\&E International, vol. 43, no. 2, pp. 123-133, 2010.

[32] A. Velichko and P. Wilcox, "Post-processing of guided wave array data for high resolution pipe inspection," Journal of the Acoustical Society of America, vol. 126, no. 6, pp. 2973-2982, 2009.

[33] A. Velichko and P. Wilcox, "A generalized approach for efficient finite element modeling of elastodynamic scattering in two and three dimensions," Journal of the Acoustical Society of America, vol. 128, no. 3, pp. 1004-1014, 2010.

[34] A. Velichko and P. Wilcox, "Efficient finite elment modeling of elastodynamic scattering from near surface and surfacebreaking defects," in AIP Conf. Proc. 1335, 59 (2011); doi: 10.1063/1.3591840, 2011.

[35] A. Velichko and P. Wilcox, "Efficient finite element modeling of elastodynamic scattering with non-reflective boundary conditions," in AIP Conf. Proc. 1430, 142 (2012); doi: 10.1063/1.4716224, 2012.

[36] L. Moreau, A. Velichko and P. Wilcox, "Accurate finite element modeling of guided wave scattering from irregular defects," NDT\&E International, vol. 45, pp. 46-54, 2012.

[37] L. Moreau, A. Hunter, A. Velichko and P. Wilcox, "3-D reconstruction f sub-wavelength scatterers from the measurement of scattered fields in elastic waveguides," IEEE Transactions on Ultrasonics, Ferroelectrics, and Frequency Control, vol. 61, no. 11, pp. 1864-1879, 2014.

[38] K. F. Graff, Wave motion in elastic solids, New York: Dover publications, INC., 1991. 
[39] J. L. Rose, Ultrasonic Waves in Solid Media, Cambridge: Cambridge University Press, 1999.

[40] B. Lin, A. Kamal, V. Giurgiutiu and T. Kamas, "Multimodal Lamb Waves Power and Transfer Function Analysis of Structurally-bounded PWAS," in ASME 2012 SMASIS, Sone Mountain, Geogia, USA, 2012.

[41] Y. Shen and V. Giurgiutiu, "Effective non-reflective boundary for Lamb waves: Theory, finite element implementation, and applications," Wave Motion, vol. 58, pp. 22-41, 2015. 